\title{
A Uniqueness Condition for Gibbs Measures, with Application to the 2-Dimensional Ising Antiferromagnet
}

\author{
J. van den Berg * \\ CWI, Kruislaan 413, NL-1098 SJ Amsterdam, The Netherlands; e-mail jvdberg@cwi.nl
}

Received February 4, 1992; in revised form August 28, 1992

\begin{abstract}
A uniqueness condition for Gibbs measures is given. This condition is stated in terms of (absence of) a certain type of percolation involving two independent realisations. This result can be applied in certain concrete situations by comparison with "ordinary" percolation. In this way we prove that the Ising antiferromagnet on a square lattice has a unique Gibbs measure if $\beta(4-|h|)<\frac{1}{2} \ln \left(P_{c} /\left(1-P_{c}\right)\right)$, where $h$ denotes the external magnetic field, $\beta$ the inverse temperature, and $P_{c}$ the critical probability for site percolation on that lattice. Since $P_{c}$ is larger than $\frac{1}{2}$, this extends a result by Dobrushin, Kolafa and Shlosman (whose proof was computer-assisted).
\end{abstract}

\section{Introduction and General Theorem}

Our main theorem requires hardly any prerequisites and we hope the following introduction makes it also accessible to non-experts.

Let the graph $G$ be connected, countably infinite, and locally finite (the last means that each vertex has finitely many edges). The set of vertices of $G$ is denoted by $V_{G}$. Vertices will typically be denoted by $i, j, v, w$ etc., possibly with a subscript. Two vertices $v$ and $w$ are said to be adjacent, or neighbours (notation: $v \sim w$ ) if there is an edge between them.

A path from $v$ to $w$ is a sequence of vertices $v_{1}=v, v_{2}, \ldots, v_{l}=w$ with the property that consecutive vertices are adjacent. An infinite path is a sequence $v_{1}, v_{2}, \ldots$ with the property that consecutive vertices are adjacent, and which contains infinitely many different vertices.

For $B \subset V_{G}$, $\delta_{B}$ will denote the boundary of $B$, i.e. the set of all vertices which are not in $B$ but adjacent to some vertex in $B$.

\footnotetext{
* The research which led to this paper started while the author was at Cornell University, partly supported by the U.S. Army Research Office through the Mathematical Sciences Institute of Cornell University
} 
Percolation. Suppose each vertex $i$ is, independent of all other vertices, open (i.e. accessible) with probability $p_{i}$ and closed with probability $1-p_{i}$. Denote the corresponding probability measure by $P_{\left\{p_{\imath}\right\}}$. For a realisation of the process a path is called open if all its vertices are open. We say that percolation occurs if $P_{\left\{p_{\imath}\right\}}$ (there exists an infinite open path) $>0$ (in which case this probability is even 1 since the event is a tail event). In case all $p_{i}$ 's are equal, say $p$, we write $P_{p}$ for the above probability measure and define the critical probability $p_{c}=\inf \left\{p: P_{p}\right.$ (there exists an infinite open path) $\left.>0\right\}$. This critical probability depends on $G$. One of the first results in percolation was to show that $p_{c}<1$ for a large class of graphs, including the square lattice [Broadbent and Hammersley (1957)]. The above model is called independent site percolation. If the vertices do not behave independent of each other we speak of dependent percolation and if the edges rather than the vertices are open or closed we speak of bond percolation. For further study, see Grimmett (1989) and Kesten (1982).

Markov Fields and Gibbs Measures. Let $S$ be a finite or countably infinite set and define $\Omega=S^{V_{G}}$. Elements of $\Omega$ will typically be denoted by $\omega \equiv\left(\omega_{\imath}, i \in V_{G}\right)$. We are interested in certain probability measures $\mu$ on $\Omega$ (equipped with the $\sigma$-algebra generated by $\left(\omega_{i}=s\right), i \in V_{G}, s \in S$; we will call this the obvious $\sigma$-algebra). Roughly speaking, $\mu$ is called a Markov field if, for each finite set of vertices $B$, the conditional distribution of the configuration inside $B$, given the configuration outside $B$, depends only on the configuration on $\delta_{B}$. Such a set of conditional probabilities, indexed by the finite sets $B$, the configurations on $B$, and the configurations on $\delta_{B}$, is called a specification of $\mu$. There may be more Markov fields with the same specification. They are called its Gibbs measures. For more general and precise definitions see Georgii (1988) and Prum and Fort (1991). An intuitively appealing introduction is Kindermann and Snell (1980). A central problem in the theory is to determine whether a given specification has a unique Gibbs measure. In case of nonuniqueness we say that there is a phase transition. The most well-known condition which implies uniqueness is Dobrushin's condition of weak dependence [Dobrushin (1968a)]. For references to other uniqueness results see the bibliographical notes for Chap. 8 of Georgii (1988). In this paper we prove a uniqueness condition involving two independent realisations. To state our result we need another definition. Let $\omega$ and $\omega^{\prime}$ be two realisations. A path of disagreement for the pair $\left(\omega, \omega^{\prime}\right)$ is a path in $G$ on which all vertices $i$ have $\omega_{i} \neq \omega_{i}^{\prime}$.

Theorem 1. Let $G$ be a countable, locally finite, connected graph, $V_{G}$ its set of vertices, $S$ a finite or countably infinite set, and $\Omega=S^{V_{G}}$. Let the probability measures $\mu$ and $\mu^{\prime}$ on $\Omega$ (with the obvious $\sigma$-algebra) be Markov fields with the same specification. Consider two independent realisations, one under $\mu$, the other under $\mu^{\prime}$. If $\left(\mu \times \mu^{\prime}\right)\left(\left(\omega, \omega^{\prime}\right)\right.$ has an infinite path of disagreement $)=0$, then $\mu=\mu^{\prime}$.

Remarks. (i) The reverse is obviously false: For example, let $G$ be a graph whose critical probability $p_{c}$ (for site percolation) is strictly smaller than 1 , and let $S=$ $\{1, \ldots, n\}$. Let $\mu$ be the probability measure under which each $i \in V_{G}$, independent of all other vertices, is in state $s \in S$ with probability $1 / n$. Uniqueness is trivial in this case. However, if $\omega$ and $\omega^{\prime}$ are two independent realisations of this process, then the process $\left(I\left(\omega_{i} \neq \omega_{i}^{\prime}\right)\right)_{i \in V_{G}}$, where $I(\cdot)$ denotes the indicator function, is i.i.d. with parameter $p=1-1 / n$. Hence, if $n$ is taken sufficiently large, then $p>p_{c}$ and we have, with probability 1 , an infinite path of disagreement. This example shows that 
our theorem is, in certain cases, completely useless. However, in some other cases it is quite powerful, as the application in Sect. 2 shows.

(ii) Methods involving two independent realisations have been used, with much success, earlier in this field [see e.g. Lebowitz (1974), Percus (1975), and Aizenman (1980)].

(iii) It seems natural to expect that improved results may be obtained by taking a more complicated coupling (instead of independent realisations). This will be the subject in van den Berg and Maes (1992).

Proof of Theorem 1. Suppose the assumption holds, i.e. $\left(\mu \times \mu^{\prime}\right)\left(\left(\omega, \omega^{\prime}\right)\right.$ has an infinite path of disagreement $)=0$. Let $A$ be an arbitrary finite set of vertices, and $s_{1}, s_{2}, \ldots, s_{|A|}$ an arbitrary sequence of elements of $S$ (where $|A|$ denotes the number of elements of $A$ ). Further, let $E$ be the event $\left\{\omega \in S^{V}: \omega_{i}=s_{i}\right.$ for all $\left.i \in A\right\}$. We have to prove that $\mu(E)=\mu^{\prime}(E)$. For each pair $\left(\omega, \omega^{\prime}\right)$ the cluster of disagreement containing $A$ is defined as the subset $C_{A}$ of $V_{G}$ which consists of $A$ as well as all vertices $i$ for which there exists a path of disagreement to some vertex in $\delta_{A}$. Let $T: \Omega \times \Omega \rightarrow \Omega \times \Omega$ be the transformation which exchanges $\omega$ and $\omega^{\prime}$ on the above cluster of disagreement. More precisely, $T\left(\omega, \omega^{\prime}\right)=\left(\sigma, \sigma^{\prime}\right)$, where $\sigma_{i}$ equals $\omega_{i}^{\prime}$ if $i \in C_{A}$ and equals $\omega_{\imath}$ otherwise, and, similarly, $\sigma_{i}^{\prime}$ equals $\omega_{i}$ if $i \in C_{A}$ and $\omega_{i}^{\prime}$ otherwise. This transformation is obviously $1-1$. Moreover, $C_{A}$ is finite with probability one (by assumption). From this, together with the Markov property of $\mu$ and $\mu^{\prime}$, and the fact that $\mu$ and $\mu^{\prime}$ have the same specification, it is quite easy to see that $T$ is also measure preserving: sum over all possibilities for $C_{A}$, and all possible configurations on $C_{A} \cup \delta C_{A}$. (This way of using the Markov property is somewhat similar to that in Russo (1979.) Hence, since $E$ involves only vertices of $A$ (which is by definition contained in $C_{A}$ ), we have

$$
\mu(E)=\left(\mu \times \mu^{\prime}\right)(E \times \Omega)=\left(\mu \times \mu^{\prime}\right)(T(E \times \Omega))=\left(\mu \times \mu^{\prime}\right)(\Omega \times E)=\mu^{\prime}(E),
$$

which completes the proof.

The condition in Theorem 1 involves dependent site percolation. In certain situations it is useful to compare this process with independent site percolation:

Corollary 1. Let $G, S, \mu$ and $\mu^{\prime}$ be as defined in Theorem 1. Consider again two independent realisations, one under $\mu$, the other under $\mu^{\prime}$. Let, for each vertex $i, N_{\imath}$ be the set of neighbours of $i$, and define

$$
p_{\imath}=\sup _{\alpha, \alpha^{\prime} \in S^{N_{i}}}\left(\mu \times \mu^{\prime}\right)\left(\omega_{\imath} \neq \omega_{i}^{\prime} \mid \omega_{j}=\alpha_{j} \text { and } \omega_{j}^{\prime}=\alpha_{j}^{\prime} \text { for all } j \in N_{i}\right) .
$$

Consider the percolation process where each vertex of $G$, independently of all others, is open with probability $p_{i}$ and closed with probability $1-p_{i}$. If $P_{\left\{p_{\imath}\right\}}$ (there exists an infinite open path) $=0$, then $\mu=\mu^{\prime}$.

Remark. It is clear from the definition of the $p_{i}$ 's in (1) that this corollary can easily be reformulated as a uniqueness condition for (Markovian) specifications.

Proof of Corollary 1. Let, for each $i, \mathfrak{I}_{i}$ be the $\sigma$-field generated by the random variables $\omega_{\jmath}, j \neq i$ and the random variables $\omega_{j}^{\prime}, j \neq i$. Since $\mu$ and $\mu^{\prime}$ are Markov fields it is obvious that $\left(\mu \times \mu^{\prime}\right)\left(\omega_{\imath} \neq \omega_{i}^{\prime} \mid \Im_{i}\right)$ is, a.s., at most $p_{i}$. Therefore it is intuitively obvious that the process $\left(I\left(\omega_{\imath} \neq \omega_{i}^{\prime}\right)\right)_{i \in V_{G}}$ is stochastically dominated by the process (I(vertex $i$ is open) $)_{\imath \in V_{G}}$. [This can be easily proved by standard arguments as, e.g., in the proof of Lemma 1 in Grimmett and Marstrand (1990)]. 
In particular, the probability that $\left(\omega, \omega^{\prime}\right)$ has an infinite path of disagreement is smaller than or equal to $P_{\left\{p_{2}\right\}}$ (there exists an infinite open cluster). Now apply Theorem 1.

\section{Application: Phase Diagram of the 2-Dimensional Ising Antiferromagnet}

In this section $G$ is the square lattice, i.e. the lattice whose vertices are the elements of $Z^{2}$, and where two vertices $v=\left(v_{1}, v_{2}\right)$ and $w=\left(w_{1}, w_{2}\right)$ have an edge in between iff $\left|v_{1}-w_{1}\right|+\left|v_{2}-w_{2}\right|=1$. The Ising antiferromagnet has two parameters, the external magnetic field $h$ and the temperature $T$ (or, instead, the inverse temperature $\left.\beta=\frac{1}{T}\right)$. Each vertex $i$ can have spin $\omega_{\imath}=+1$ or -1 , i.e. $S=\{-1,+1\}$. Its Hamiltonian is given by

$$
H=\sum_{i \sim j} \omega_{i} \omega_{j}-h \sum_{j} \omega_{j}
$$

This means that we are dealing with Markov fields with the property that the conditional probability that a finite set $B \subset V_{G}$ has configuration $\sigma$, given the event that its boundary has configuration $\alpha$ is proportional to

$$
\exp \left(-\beta\left(\sum_{i \sim j ; i, j \in B} \sigma_{\imath} \sigma_{\jmath}+\sum_{i \sim j ; i \in B, j \in \delta_{B}} \sigma_{i} \alpha_{j}-h \sum_{j \in B} \sigma_{j}\right)\right) .
$$

It is a standard result that at least one such probability measure exists. It has been proved by Dobrushin (1968b) that there is more than one Gibbs measure in the region $\beta(4-|h|)>\bar{u}$ with $\bar{u}$ a positive constant.

In a paper by Dobrushin, Kolafa and Shlosman (1985) the phase diagram near the points $h= \pm 4, T=0$ has been investigated. In particular, they were interested in the question whether there could be more that one Gibbs measure if $h= \pm 4$ and $T$ is sufficiently small. The main result in their paper, Theorem 2 below, shows the answer is negative (which had been made plausible before, but no rigorous proof existed).

Theorem 2 [Dobrushin, Kolafa, Shlosman (1985)]. There exist $\theta, \pi>\theta>\pi / 2$, and $r>0$ such that there is a unique Gibbs measure of the antiferromagnet on a square lattice with parameters $(h, T)$ in the domain $\left\{(h, T): h-4=r^{\prime} \cos \theta^{\prime}, T=r^{\prime} \sin \theta^{\prime}\right.$, $\left.0 \leq \theta^{\prime} \leq \theta, 0 \leq r^{\prime} \leq r\right\}$. By symmetry, a similar result holds near the points $h=-4$, $T=0$.

The proof of the theorem above is computer-assisted and based on a constructive uniqueness criterion by Dobrushin and Shlosman (1985). This criterion is of the form: "if the Gibbs specification is such that a condition $C_{V}$ is true for a finite volume $V$, then there is a unique Gibbs measure." The values of $\theta$ and $r$ which can be obtained from the paper are very close to $\pi / 2$ and 0 respectively, but it is believed that, in principle, by checking sufficiently large boxes, uniqueness for this antiferromagnet can be proved with their method whenever it holds. However, in practice the possibilities are, of course, limited by computer power.

We will show that the corollary in Sect. 1, combined with the following result on independent site percolation, yields, quite easily, a result which is stronger than Theorem 2. 
Lemma 1. Let $P_{c}$ denote the critical probability for site percolation on the square lattice.

(a) [Harris (1960)] $P_{c} \geq 1 / 2$.

(b) [Higuchi (1982)] $P_{c}>1 / 2$.

Remark. Part (a) of the above lemma was proved by Harris for bond percolation on the square lattice, but extends to site percolation [see Fisher (1961) and Hammersley (1961)].

Theorem 3. For $\beta(4-|h|)<\frac{1}{2} \ln \left(P_{c} /\left(1-P_{c}\right)\right)$ the Ising antiferromagnet on the square lattice has a unique Gibbs measure. Here $P_{c}$ denotes the critical probability for site percolation on that lattice.

Proof of Theorem 3. As remarked before, the existence of at least one Gibbs measure is a standard result. By symmetry we may restrict to the case $h>0$. We apply the corollary in Sect. 1. In this model $p_{i}$, defined in (1), does not depend on $i$ so we omit the subscript. By taking $B$ the set consisting of just one element, say the origin, and noting that the conditional distribution of the spin at the origin, given the spins of its four neighbours, is a function of the sum $n$ of those neighbour spins, it takes only a few elementary steps to derive from (3) that

$$
\frac{p}{1-p}=\max _{-4 \leq n^{\prime} \leq n \leq 4} \frac{\cosh \left(\beta\left(n-n^{\prime}\right)\right)}{\cosh \left(\beta\left(2 h-n-n^{\prime}\right)\right)} .
$$

Now set

$$
h=4+\frac{u}{\beta} .
$$

The condition in the theorem can now be written as

$$
u>-\frac{1}{2} \ln \left(P_{c} /\left(1-P_{c}\right)\right) \text {. }
$$

We have to show that, under (6), $p<P_{c}$, or, equivalently, $p /(1-p)<P_{c} /\left(1-P_{c}\right)$. First, we can now write (4) as

$$
\frac{p}{1-p}=\max _{-4 \leq n^{\prime} \leq n \leq 4} \frac{\cosh \left(3\left(11-n^{\prime}\right)\right)}{\cosh \left(\beta\left(8-n-n^{\prime}\right)+2 u\right)} .
$$

Now note that $0 \leq n-n^{\prime} \leq 8-n-n^{\prime}$ and use that the function $x \rightarrow \cosh (x)$ is increasing for $x \geq 0$. In case $u=0$ this gives immediately $p /(1-p) \leq 1$, which, combined with (6), yields the desired inequality. In case $u>0$, use again the monotonicity of $\cosh (x)$ to obtain $p /(1-p)<1$, which, combined with part (a) of the lemma, yields again $p<P_{c}$. Finally, as to the case $u<0$, note that the value of the denominator in the right-hand side of (7) for that case is larger than $\exp (2 u)$ times the corresponding value for the case $u=0$. Hence, the value of $p /(1-p)$ at $u<0$ is smaller than $\exp (-2 u)$ times its value at $u=0$. In other words, if $u<0$, then $p /(1-p)<\exp (-2 u)$, which by (6) is smaller than $P_{c} /\left(1-P_{c}\right)$.

Remarks. (a) Note that the above theorem combined with part (a) of Lemma 1 implies uniqueness whenever $|h|>4$. With the strong inequality in part (b) of the lemma it clearly implies (and extends) the result by Dobrushin, Kolafa and Shlosman stated in Theorem 2 above. Using better lower bounds for $P_{c}$, our theorem yields automatically stronger uniqueness results. For instance, Toth (1985) has proved $P_{c}>0.503 \ldots$.. This bound has been further improved by Menshikov and Pelikh (1989). 
(b) Intimately connected with Theorem 2 is the result (by Dobrushin et al. in the same paper) that the critical activity $a_{c}$ for the hard-square lattice gas model is larger than 1. Our method easily yields $a_{c} \geq P_{c} /\left(1-P_{c}\right)$. This and other new rigorous results for hard-core lattice gas models are given in van den Berg and Steif (1992).

(c) In this paper we have restricted to mathematically rigorous results, and we have not mentioned the many detailed results for the Ising antiferromagnet which have been obtained by interesting but non-rigorous methods [see e.g. Blöte and Wu (1990)].

Acknowledgements. I thank R. M. Burton, P. W. Kasteleyn, and J. E. Steif for stimulating discussions.

\section{References}

1. Aizenman, M.: Translation invariance and instability of phase coexistence in the two-dimensional Ising system. Commun. Math. Phys. 73, 83-94 (1980)

2. Berg, J., van den, Steif, J.E.: On the hard-core lattice gas model, percolation and certain loss networks. Preprint (1992)

3. Berg, J., van den, Maes, C.: Preprint (1992)

4. Blöte, H.W.J., Wu, X.-N.: Accurate determination of the critical line of the square Ising antiferromagnet in a field. J. Phys. A: Math. Gen. 23, L 627-L 631 (1990)

5. Broadbent, S.R., Hammersley, J.M.: Percolation processes. I. Crystals and mazes. Proceedings of the Cambridge Philosophical Society 53, 629-641 (1957)

6. Dobrushin, R.L.: The description of a random field by means of conditional probabilities and conditions of its regularity. Theor. Prob. Appl. 13, 197-224 (1968a)

7. Dobrushin, R.L.: The problem of uniqueness of a Gibbs random field and the problem of phase transition. Funct. Anal. Appl. 2, 302-312 (1968b)

8. Dobrushin, R.L., Shlosman, S.B.: Constructive criterion for the uniqueness of a Gibbs field. In: Fritz, J., Jaffe, A., Szász, D. (eds.), Statistical mechanics and dynamical systems. Boston: Birkhäuser 1985, pp. 371-403

9. Dobrushin, R.L., Kolafa, J., Shlosman, S.B.: Phase diagram of the two-dimensional Ising antiferromagnetic (computer-assisted proof). Commun. Math. Phys. 102, 89-103 (1985)

10. Fisher, M.E.: Critical probabilities for cluster size and percolation problems. J. Math. Phys. 2, 620-627 (1961)

11. Georgii, H.-O.: Gibbs measures and phase transitions. Berlin, New York: de Gruyter 1988

12. Grimmett, G.R.: Percolation. Berlin, Heidelberg, New York: Springer 1989

13. Grimmett, G.R., Marstrand, J.M.: The supercritical phase of percolation is well behaved. Proc. Roy. Soc. London Ser. A 430, 439-457 (1990)

14. Hammersley, J.M.: Comparison of atom and bond percolation. J. Math. Phys. 2, 728-733 (1961)

15. Harris, T.E.: A lower bound for the critical probability in a certain percolation process. Proc. Cambridge Phil. Soc. 56, 13-20 (1960)

16. Higuchi, Y.: Coexistence of the infinite $\left(^{*}\right)$ clusters: a remark on the square lattice site percolation. ZfW 61, 75-81 (1982)

17. Kesten, H.: Percolation theory for mathematicians. Boston: Birkhäuser 1982

18. Kindermann, R., Snell, J.L.: Markov random fields and their applications. Contemporary Mathematics, Vol. 1. Providence, R.I.: Amer. Math. Soc. 1980

19. Lebowitz, J.L.: GHS and other inequalities. Commun. Math. Phys. 35, 87-92 (1974)

20. Menshikov, M.V., Pelikh, K.D.: Matematicheskie Zametki 46, 38-47 (1989)

21. Percus, J.: Correlation inequalities for Ising spin lattices. Commun. Math. Phys. 40, 283-308 (1975)

22. Prum, B., Fort, J.C.: Stochastic processes on a lattice and Gibbs measures. Dordrecht, Boston, London: Kluwer 1991

23. Russo, L.: The infinite cluster method in the two-dimensional Ising model. Commun. Math. Phys. 67, 251-266 (1979)

24. Toth, B.: A lower bound for the critical probability of the square lattice site percolation. ZfW 69, 19-22 (1985) 